\title{
Sandawe Language
}

National Cancer Institute

\section{Source}

National Cancer Institute. Sandawe Language. NCI Thesaurus. Code C154103.

A click language spoken by the Sandawe people in the Dodoma region of Tanzania. 